\title{
The effect of initial reward on the rate of reacquisition
}

I-NING HUANG, Wisconsin State University, Whitewater, Wis. 53190, and HWAI-HUEY HUANG, University of Texas, Austin, Tex. 78712

A 2 by 2 factorial design, using 40 rats in a runway, was employed to investigate the effect of initial reward on the rate of reacquisition following an interpolated nonreward training. The reacquisition rates were found to be significantly faster for the groups when the reward was the same in the initial and reacquisition phases (transfer shift) than when it was different (nontransfer shift). In the nontransfer shift condition, there was a tendency for the group with a gain in stimulus intensity $(V)$ to adjust the reacquisition asymptotic level faster than did the group with a loss in $V$.

Recent experimental findings have shown that a previously established S-R connection is not directly affected under some subsequent interpolated training. Specifically, a depression effect occurred despite an intervening nonreward training prior to the reward shift phase (Vogel, Mikulka, \& Spear, 1966), and a rapid reacquisition rate was exhibited when the previous reward condition was reinstated in spite of an interpolated nonreward (Huang, 1969; Logan, 1960), different reward magnitude (Capaldi \& Lynch, 1967; Huang, 1969; Wagner \& Thomas, 1966), or even a qualitatively different reward training (Huang, 1969).

The findings seem to suggest that the change of response strength during subsequent training may be attributed essentially to a change of the stimulus complex, and consequently a process of generalization decrement and change of stimulus intensity (V), from the original to the subsequent training. Since the original stimulus complex controlling an organism's behavior was assumed to be merely replaced by the stimulus complex of the subsequent training condition, the previously learned S-R connection was not directly affected. Therefore, a rapid rate of reacquisition was indeed expected when the original reward condition was reinstated. Furthermore, two specific and interesting predictions can be deduced and tested from this line of theorizing: (1) The reacquisition rate would be faster for the condition in which the reward was the same in the original and in reacquisition training (transfer shift). than if it was different (nontransfer shift), because the latter suffers from a reduction in response strength due to generalization decrement and possibly a weak V. (2) Under the nontransfer shift condition, provided with equal generalization decrement, the reacquisition rate would be slower for the one with a loss in $\mathrm{V}$ than for one without loss or with a gain in $\mathrm{V}$.

\section{SUBJECTS}

Forty naive male albino rats, approximately $175-200 \mathrm{~g}$ in weight and about 70 days old on arrival at the laboratory, were purchased from Sprague-Dawley, Madison, Wisconsin. APPARATUS

The apparatus consisted of a mid-gray wooden runway, $84 \times 3 \frac{1}{2} \times 9$ in. high, covered with $1 / 4$-in. hardware cloth. It had three basic sections: start, 12 in.; run, 60 in.; and an L-shape goal, 12 in. The start and goal sections were separated from the run by a Hunter double-door gate and a Hunter single-door gate, respectively. Response time was measured by a Standard electric stopclock in $.01-\mathrm{sec}$ intervais, from the opening of the clear door of the starting gate until $S$ interrupted a photobeam 6 in. before the second gate. The runway was uniformly illuminated by a 20-W illuminator hanging approximately $4 \mathrm{ft}$ above the run section.

\section{PRELIMINARY TRAINING}

On arrival (Day 1) at the laboratory, Ss were housed in individual cages. They were put on a 10-g Lab Chow with ad lib water maintenance schedule that continued throughout the experiment. On Days 5-8, all $\mathrm{Ss}$ were handled individually for $3 \mathrm{~min}$ before the daily feeding. The Ss were given $10.045 \mathrm{~g}$ Noyes pellets in their home cages on Day 8. On Day 9, each $S$ was allowed 1 to $2 \mathrm{~min}$ to explore the goal section, with five pellets in the metal food tray.

\section{EXPERIMENTAL TRAINING}

The experimental training was divided into three phases: Phase 1,30 days (Days 10-39); Phase 2, 33 days (Days 40-72); and Phase 3, 16 days (Days 73-88). All Ss were run at one trial a day. At the beginning of experimental training, the Ss were assigned randomly to one of the four groups: SNS, SNL, LNS, and LNL. Group designations are given in terms of the magnitude of the reward (large reward, L; small reward, S; nonreward, $N$ ) received in each phase; i.e., SNL means a group received small reward, nonreward, and large reward in Phases 1,2, and 3 , respectively. Since the initial (Phase 1) and the reacquisition (Phase 3) reward conditions were each varied in two levels, large vs small, forming a 2 by 2 factorial design, the effect of initial reward on the rate of reacquisition may be evaluated by comparing the performance among the groups in Phase 3. A trial was begun by removing $S$ from the home cage and placing him in the startbox. Two to 5 sec later, E opened the black door of the starting gate, so that the $S$ could see the runway through the clear door of the gate. When the $S$ oriented toward the runway, $E$ opened the clear door and started the stopclock. As soon as the $S$ interrupted the photobeam, stopping the clock, and entered the goal section, the door separating the run and goal sections was lowered to prevent retracing. Immediately after the consumption of either one $.045-\mathrm{g}$ Noyes pellet (S) or 10 pellets (L), $S$ was retumed to the home cage. On a nonrewarded trial, $S$ was confined in the goal section for $30 \mathrm{sec}$. If $S$ failed to enter the goal section within $30 \mathrm{sec}$ on a trial, a response time of $30 \mathrm{sec}$ was assigned and the $\mathrm{S}$ was removed and returned to the home cage. Ss were given the daily feeding at least $15 \mathrm{~min}$ after the trial.

\section{RESULTS}

The response times of the four groups during the three phases of training were converted into speed measures (feet/second) and are presented in Fig. 1.

Unless otherwise noted, each point on the graph represents a group mean of 10 $\mathrm{Ss}$ ' medians of a block of three trials. Since the stimulus complex controlling the S's behavior on the $n^{\text {th }}$ trial in the runway was assumed to be composed of the outcomes that occurred on the $n-1^{\text {th }}$ trial and the environmental stimuli present on Trial $n$, Days 11-40, Days 41-73, and Days 74-88 of training constituted the data of Phases 1,2 , and 3 , respectively.

Differences between the four groups on the initial experimental training trial (Day 10; A, on Fig. 1) was insignificant $(F<1)$. The response speeds of large (LNL and LNS) and small (SNL and SNS) reward conditions gradually separated and became stable as a function of further Phase 1 training trials. An analysis of variance performed on the last four trials of Phase 1 indicated that only the response speed of large reward was significantly faster than that of small reward $[F(1,36)=12.870$, $\mathrm{p}<.011$, with the trial effect and reward conditions by trial effects insignificant (all Fs $<1$ ).

One essential purpose of the Phase 2 training was to adjust the behavior of the Ss at a comparable level and to provide the potentiality for measure before the critical and third phase was introduced. The differences of speeds between the four groups at the 11th and last block of 


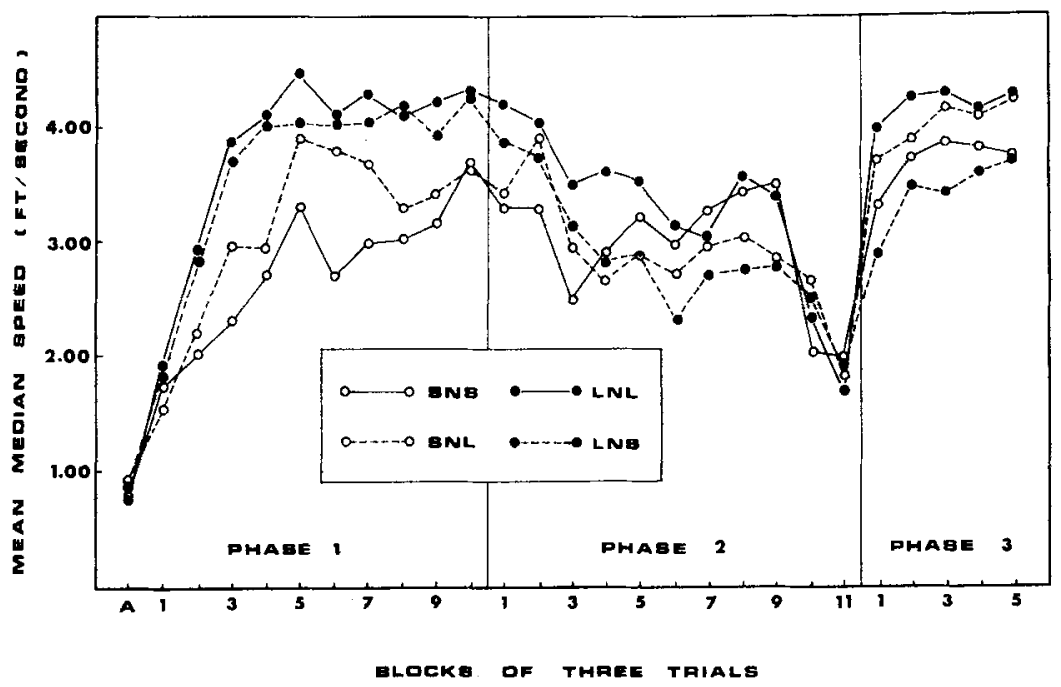

Phase 2 were eventually negligible $(F<1)$; the response speeds were $1.91,1.67,1.99$, and $1.84 \mathrm{ft} / \mathrm{sec}$ for LNS, LNL, SNS, and SNL, respectively.

In Phase 3 , the reacquisition speed was faster for the current large reward than for the samll-reward condition $[\mathrm{F}(1,36)=27.532, \mathrm{p}<.01]$. The Phase-1-reward-magnitude effect on reacquisition was insignificant, $\mathrm{F}<\mathrm{l}$. Interestingly, the interaction effect of Phase 1 Reward Magnitude by Phase 3 Reward Magnitude was significant $[F(1,36)=5.990, p<.05]$. That is, the reacquisition speed was significantly faster for transfer shift groups (LNL and SNS) than for nontransfer shift groups (LNS and SNL). Considering within-Ss variation in Phase 3 , only the block effect was significant $[\mathrm{F}(4,144)=18.886, \mathrm{p}<.01]$, with all Groups by Blocks interaction terms insignificant (all ps $>.05$ ).

As shown in Fig. 1, the speed that LNS approached the performance level of SNS was slower than the speed that SNL approached the level of LNL in Phase 3. The response speed of SNL was slower than the speed of LNL on Blocks 1 $[F(1,18)=1.706, p>.05]$ and 2 $[F(1,18)=1.698, p>.05]$. The speed of LNS was also slower than that of SNS on the first two blocks $[F(1,18)=3.644$, $.05<\mathrm{p}<.10$ and $\mathrm{F}(1,18)=2.535$, $\mathrm{p}>.05$, respectively]. However, by Block 3, the response speed of SNL adjusted to a comparable level of LNL
Fig. 1. Speed on the initial (A) trial and in successive blocks of three trials for each of the four groups on the three phases of training.

in $V$ for LNS (from $V_{R L}$ to $V_{R S}$ ), while there was a gain in $V$ for SNL (from $V_{R S}$ to $V_{R L}$ ) during the moment of transition. Therefore, it would need more trials to reach the new performance asymptote to compensate for the loss of V in LNS.

The findings of rapid rates of adjustment during reacquisition in transfer shift groups and that, within the nontransfer shift groups, SNL adjusted to its asymptotic level in Phase 3 faster than did LNS suggest the following viewpoints: First, it suggests the notion of the relatively permanent effect of prior reinforcement experience that would not be unlearned or erased by an interpolated nonreward training. Second, the change of S's behavior on some subsequent situation may be attributed to a change of stimulus complex, i.e., a process of generalization decrement and change of $V$.

\section{REFERENCES}

CAPALDI, E. J. Partial reinforcement: A hypothesis of sequential effects. Psychological Review, 1966, 73, 459-477.

CAPALDI, E. J. A sequential hypothesis of instrumental learning. In K. W. Spence and J. T. Spence (Eds.), The psychology of learning and motivation: Advances in research and theory. Vol. 1. New York: Academic Press, 1967.

CAPALDI, E. J., \& LYNCH, D. Repeated shifts in reward magnitude: Evidence in favor of an associational and absolute (noncontextual) interpretation. Journal of Experimental Psychology, 1967, 75, 226-235.

HUANG, I-NING Successive contrast effects as a function of type and magnitude of reward. Joumal of Experimental Psychology, in press.

LOGAN, F. A. Incentive. New Haven: Yale University Press, 1960.

VOGEL, J. R., MIKULKA, P., \& SPEAR, N. E. Effect of interpolated extinction and level of training on the "depression effect." Journal of Experimental Psychology, 1966, 72, 51-60.

WAGNER, A. R., \& THOMAS, E. Reward magnitude shifts: A saving effect. Psychonomic Science, 1966, 4, 13-14. NOTE

1. This study was supported in part by a research grant from the Research Advisory Committee, Wisconsin State University at Whitewater, to the first author. The assistance of Roger Helmer is gratefully acknowledged. Requests for reprints should be sent to I-Ning Huang, Department of Psychology, Wisconsin State University, Whitewater, Wisconsin 53190. 